\title{
Improving gelation efficiency and cytocompatibility of visible light polymerized thiol- norbornene hydrogels via addition of soluble tyrosine
}

Han Shih, ${ }^{a}$ Hung-Yi Liu, ${ }^{a}$ and Chien-Chi Lin ${ }^{\mathrm{a}, \mathrm{b}^{*}}$

${ }^{a}$ Weldon School of Biomedical Engineering, Purdue University, West Lafayette, IN, USA.

${ }^{b}$ Department of Biomedical Engineering, Indiana University-Purdue University Indianapolis, Indianapolis, IN, USA

*To whom correspondence should be made:

Prof. Chien-Chi Lin

Associate Professor of Biomedical Engineering

Indiana University-Purdue University Indianapolis

723 W. Michigan St. SL220K

Indianapolis, IN 46202

Phone: (317)274-0760

Email: lincc@iupui.edu

Key Words: Visible light, photopolymerization, thiol-ene hydrogel, PEG, tyrosine

This is the author's manuscript of the article published in final edited form as:

Shih, H., Liu, H. Y., \& Lin, C. C. (2017). Improving gelation efficiency and cytocompatibility of visible light polymerized thiol-norbornene hydrogels via addition of soluble tyrosine. Biomaterials Science, 5(3), 589-599. http://dx.doi.org/10.1039/C6BM00778C 


\section{ABSTRACT}

Hydrogels immobilized with biomimetic peptides have been used widely for tissue engineering and drug delivery applications. Photopolymerization has been among the most commonly used techniques to fabricate peptide-immobilized hydrogels as it offers rapid and robust peptide immobilization within a crosslinked hydrogel network. Both chain-growth and stepgrowth photopolymerizations can be used to immobilized peptides within covalently crosslinked hydrogels. Previously developed visible light mediated step-growth thiol-norbornene gelation scheme has demonstrated efficient crosslinking of hydrogels composed of inert poly(ethylene glycol)-norbornene (PEGNB) macromer and small molecular weight bis-thiol linker, such as dithiothreitol (DTT). Compared with conventional visible light mediated chain-polymerizations where multiple initiator components are required, step-growth photopolymerized thiol-norbornene hydrogels are more cytocompatible for in situ encapsulation of radical sensitive cells (e.g., pancreatic $\beta$-cells). This contribution explored visible light based crosslinking of various biscysteine containing peptides with macromer 8-arm PEGNB to form biomimetic hydrogels suitable for in situ cell encapsulation. It was found that the addition of soluble tyrosine during polymerization not only significantly accelerated gelation, but also improved the crosslinking efficiency of PEG-peptide hydrogels as evidenced by a decreased gel point and enhanced gel modulus. In addition, soluble tyrosine drastically enhanced the cytocompatibility of the resulting PEG-peptide hydrogels, as demonstrated by in situ encapsulation and culture of pancreatic MIN6 $\beta$-cells. This visible light based thiol-norbornene crosslinking mechanism provides an attractive gelation method for preparing cytocompatible PEG-peptide hydrogels for tissue engineering applications. 


\section{INTRODUCTION}

Bioactive or biomimetic hydrogels suitable for drug delivery and tissue engineering applications can be fabricated from naturally derived materials such as alginate [1], glycosaminoglycans [2-5], fibrin [6, 7], chitosan [8-10], dextran [11], collagen [12], and gelatin [13, 14]. Although hydrogels derived from natural matrices are inherently bioactive, it remains challenging in tuning their bioactivity, mechanics, and degradation features [15]. On the other hand, hydrogels prepared from purely synthetic polymers, such as poly(ethylene glycol) (PEG) and poly(vinyl alcohol) (PVA), have excellent tunability in material mechanical properties but they do not possess bioactive features necessary for promoting cell viability and function [16-18]. Hence, biomimetic peptides are routinely used to render the otherwise inert synthetic hydrogel bioactive. A classical way of immobilizing peptides in synthetic hydrogel is through chain-growth copolymerization of (meth)acrylated macromers and (meth)acrylated peptides [19]. Alternatively, cysteine containing peptides could be readily crosslinked with acrylated macromers through a mixed-mode thiol-acrylate polymerization [20]. For example, Lin and Anseth incorporated cysteine-bearing glucagon-like peptide 1 (GLP-1C) into PEG-diacrylate (PEGDA) hydrogel to improve the viability and insulin secretion of encapsulated islets [21]. PEG-peptide hydrogel could also be polymerized via Michael-type addition between nucleophilic macromers (e.g., acrylate, maleimide, or vinyl sulfone) and cysteine-containing peptides [22-25]. For example, Garcia and colleagues synthesized bioactive PEG-peptide hydrogels using 4-arm PEG-maleimide with cysteine-containing protease-sensitive and integrin-binding peptides (i.e., CRGDS) to promote the performance of encapsulated islets in vivo [26].

While PEG-peptide hydrogels have found various successful applications, limitations and challenges exist. In particular, chain-polymerized hydrogels are known to form network containing heterogeneous and hydrophobic polyacrylate kinetic chains and high molecular weight degradation products [27-30]. On the other hand, the gelation kinetics of Michael-type hydrogels 
cannot be easily controlled and the resulting gel network often contains high degrees of structural defects [31]. To overcome these issues, Anseth and colleagues developed PEG-peptide hydrogels using thiol-norbornene photo-click reaction [32]. In this system, low intensity and long wavelength ultraviolet (UV) light $\left(5-10 \mathrm{~mW} / \mathrm{cm}^{2}, 365 \mathrm{~nm}\right)$ was used to generate primary radicals from cleavage-type photoinitiators (e.g., lithium acylphosphinate or LAP) [32]. These radicals deprotonate thiol groups on cysteine residues of the peptide crosslinkers to afford thiyl radicals capable of reacting to norbornene moiety in a strictly step-growth polymerization mechanism. The advantages of step-growth thiol-norbornene reaction include rapid, ambient, and aqueous reaction conditions, as well as spatial-temporal control over gelation kinetics [33-35]. Furthermore, thiol-norbornene photo-click reaction is not susceptible to oxygen inhibition, providing rapid gelation when comparing with acrylate-based chain-growth photopolymerizations at equivalent macromer concentration and functionality [36, 37]. Thiol-norbornene photo-click reactions also limit disulfide bond formation due to radical-mediated disulfide cleavage [38], thus increasing the degree of network crosslinking and mechanical properties of the resulting hydrogels [32].

While current thiol-norbornene hydrogels have shown to be cytocompatible for mammalian cells $[32,37,39-46]$, the use of UV-light still raised concerns of DNA and protein damage [47]. In this regard, we have developed step-growth thiol-norbornene photo-click PEGbased hydrogels using a visible light source $(400 \mathrm{~nm}$ to $700 \mathrm{~nm})$ and a type II photoinitiator (e.g., eosin-Y) [48]. Mechanistically, eosin-Y could be excited by a visible light source to abstract hydrogen from thiol-containing crosslinkers, such as dithiothreitol (DTT), to form thiyl radicals. These thiyl radicals then react with the norbornene moieties on multi-arm PEG macromers to form thioether bonds and generate carbonyl radicals. Termination occurs when the carbonyl radicals abstract hydrogen from other thiol-containing molecules. This reaction scheme not only preserves all advantages offered by thiol-norbornene photopolymerization, but also eliminates the use of potentially cytotoxic components (i.e., co-initiator and co-monomer) required in the conventional 
visible light based photopolymerization. We have shown that purely synthetic hydrogels (i.e., PEGNB crosslinked with dithiothreitol, DTT) prepared through this reaction were highly cytocompatible for in situ encapsulation of pancreatic $\beta$-cells and human mesenchymal stem cells (hMSCs) [48]. In an attempt to create bioactive hydrogels using visible light thiol-norbornene photopolymerization, we replaced DTT with bis-cysteine-bearing peptides as the hydrogel crosslinker. However, gelation efficiency was influenced significantly by the sequence of the biscysteine peptides. To improve the crosslinking efficiency of visible light initiated thiol-norbornene hydrogels using bis-cysteine peptide linkers, we explored the addition of soluble amino acids capable of altering the photo-excitability of eosin-Y $[49,50]$. It was discovered that the addition of soluble tyrosine could increase the degree of cysteine deprotonation, which led to an improvement in thiol-norbornene gelation efficiency. In addition, we systematically studied the gelation kinetics and physical properties of PEG-peptide hydrogel formed by visible light initiated thiol-norbornene crosslinking in the presence of soluble tyrosine. We further evaluated the cytocompatibility of these PEG-peptide hydrogels through in situ encapsulation of both pancreatic $\beta$-cells and isolated mouse islets.

\section{MATERIALS AND METHODS}

\section{Materials}

8-arm PEG and PEG-amine were purchased from JenKem Technology USA. Linear PEG (6 kDa), 5-norbornene-2-carboxylic acid, N,N'-dicyclohexylcarbodiimide (DCC), 4-(dimethylamino) pyridine (DMAP) were obtained from Sigma-Aldrich. Fmoc-protected amino acids, N,N,N',N'-

Tetramethyl-O-(1H-benzotriazol-1-yl)uronium hexafluorophosphate $\quad$ (HBTU) and hydroxybenzotriazole (HOBT) were purchased from AnaSpec. All other chemicals were obtained from Fisher Scientific unless noted otherwise. 


\section{PEGNB synthesis}

Linear PEG-norbornene (PEGdNB, 6 kDa) and PEG-octa-norbornene (PEG8NB, 20 kDa and $40 \mathrm{kDa}$ ) were synthesized following an established protocol [33]. In brief, 5-norbornene-2carboxylic acid (5-fold excess of $\mathrm{OH}$ group) and coupling reagent DCC (2.5-fold excess of $\mathrm{OH}$ group) were added to anhydrous dichloromethane (DCM). The mixture was purged with nitrogen and stirred at room temperature for 1 hour. The resulting norbornene anhydride was filtered into an addition funnel and added slowly to a flask containing PEG, DMAP (0.5-fold of OH group), and pyridine (0.5-fold of $\mathrm{OH}$ group) dissolved in anhydrous DCM. The flask was purged with nitrogen, kept on ice and allowed to react overnight in dark. The reaction was repeated for the second time to improve the functionalization of norbornene on PEG. The product was precipitated in cold ethyl ether and collected by filtration. PEGNB product was re-dissolved in double distilled water $\left(\mathrm{ddh}_{2} \mathrm{O}\right)$ and proceed with dialysis for 2 days using slightly acidic $d_{d H_{2}} \mathrm{O}(\mathrm{pH} \mathrm{6.8)}$ to prevent ester hydrolysis of PEG8NB. The pure product was obtained from lyophilization (degree of norbornene substitution >85 \%). PEGNB: ${ }^{1} \mathrm{H}$ NMR $\left(\mathrm{CDCl}_{3}, 500 \mathrm{MHz}\right): \delta-4.25(\mathrm{~m}, 2 \mathrm{H}), 5.98(\mathrm{~m}, 1 \mathrm{H}), 6.23(\mathrm{~m}$, $1 \mathrm{H})$.

To synthesize hydrolytically stable 8-arm PEG-amide-norbornene (PEG8aNB), norbornene acid (5-fold excess to amine groups) was first activated by HBTU/HOBT (5.5-fold excess to amine group) in dimethylformamide (DMF) for 3 minutes. With nitrogen gas purging, $\mathrm{N}, \mathrm{N}$-Diisopropylethylamine (DIEA, 6-fold excess to amine group) was added to the activated norbornene acid solution and stirred for 5 minutes. The solution was added drop wise to a twoneck flask containing PEG-octa-amine in DMF. After overnight reaction at room temperature, the product was precipitated in cold ethyl ether. PEG8aNB product was dried in vacuuo, re-dissolved in water and dialyzed for 2 days. ${ }^{1} \mathrm{H}$ NMR (Bruker 500) was used to confirm the degree of PEG functionalization (>90\%).

\section{Peptide synthesis}


All peptides were synthesized in an automated microwave-assisted peptide synthesizer (CEM Liberty 1). Crude peptides were cleaved from resins using phenol, trifluoroacetic acid (TFA), triisopropylsilane (TIS), and $\mathrm{dd}_{2} \mathrm{O}$. Cleaved peptides were precipitated in cold ethyl ether, collected from centrifugation, dried in vacuuo, and purified by reverse phase HPLC (PerkinElmer Flexar system). Peptide identity was confirmed by mass spectrometry (Agilent Technologies Model 1200-6520).

\section{Hydrogel fabrication and characterization}

Step-growth thiol-norbornene hydrogels were formed by visible light mediated photo-click reactions using PEG8NB and di-cysteine peptide (i.e., Cys-Gly-Gly-Gly-Cys or CGGGC) in the presence of $0.1 \mathrm{mM}$ of eosin-Y. L-tyrosine methyl ester (i.e., soluble tyrosine) dissolved in $\mathrm{ddH}_{2} \mathrm{O}$ was added in selected formulations. The concentration of PEG8NB was adjusted based on ${ }^{1} \mathrm{H}$ NMR results to afford correct norbornene concentrations in the gelation mixtures. The precursor solution was injected in between two glass slides separated by $1 \mathrm{~mm}$ thick spacers or in $1 \mathrm{~mL}$ syringe mold with an open tip. Hydrogel crosslinking was initiated by exposing the solution to 400$700 \mathrm{~nm}$ light at 70,000 lux (equivalence of $10 \mathrm{~mW} / \mathrm{cm}^{2}$ at $550 \mathrm{~nm}$ ) for 5 minutes. To characterize gel fractions, hydrogels were immediately dried in vacuuo after gelation. Dry mass $\left(\mathrm{W}_{\mathrm{Dr}, 1}\right)$ was measured and the dried polymers were incubated in $\mathrm{ddH}_{2} \mathrm{O}$ at $37^{\circ} \mathrm{C}$ on an orbital shaker for 24 hours to remove un-crosslinked (sol fraction) species. The swollen gels were dried again in vacuuo and weighed to obtain second dried polymer weights $\left(W_{\operatorname{Dry}, 2}\right)$. Gel fractions were obtained by the ratio of the two dry masses (i.e., $W_{\text {Dry,1 }} W_{\text {Dry,2 }}$ ). In selected experiments, gels were allowed to swell in $\mathrm{pH}$ 7.4 PBS for two days prior to obtaining swollen gel weights (W $\left.\mathrm{W}_{\text {swollen }}\right)$. Hydrogel swelling ratios $(q)$ were defined as the ratio of swollen gel mass to the second dried polymer mass (i.e., $W_{\text {Swollen }} W_{\text {Dry,2) }}$.

\section{Rheometry}


In situ photo-rheometry was performed at room temperature on a Bohlin CVO 100 digital rheometer equipped with a light cure cell. A macromer solution (100 $\mu \mathrm{L})$ was placed on a quartz plate in the light cure cell and irradiated with the visible light $(400-700 \mathrm{~nm}$, 70,000 lux) through a flexible light guide. Light was turned on 30 seconds after starting time-sweep measurement (5\% strain, $1 \mathrm{~Hz}$ frequency, and a gap size of $90 \mu \mathrm{m}$ ) using a $25 \mathrm{~mm}$ parallel plate geometry. Gel point (i.e., crossover time) was determined at the time when storage modulus (G') surpassed loss modulus (G").

Oscillatory rheometry in strain-sweep mode was used to obtain elastic modulus of the hydrogel. At equilibrium swelling (after 48 hours in pH 7.4 PBS), a biopsy punch was used to remove circular gel discs $(8 \mathrm{~mm}$ in diameter) from the gel slabs. Storage moduli of the hydrogels were measured using $8 \mathrm{~mm}$ parallel plate geometry with a gap size of 750 $\mu \mathrm{m}$. The elastic moduli for each gel formulation $(\mathrm{N}=3)$ were recorded from the average of the linear viscoelastic region (LVR, linear portion of G' plotting against \% strain).

\section{Thiol and norbornene consumption}

Precursor solution containing eosin-Y (at $0.1 \mathrm{mM}$ ) and various components (i.e., PEGdNB $_{6 \mathrm{kDa}}$, L-cysteine $\mathrm{HCl}$, and L-tyrosine methyl ester) was prepared in PBS (pH 7.4) and exposed to visible light (400-700 nm, 70,000 lux). Concentrations of the components were equivalent to those used in gelation studies (final concentration of norbornene and thiol = $12 \mathrm{mM}$ ). To determine thiol-consumption, portions of non-gelling solution were collected throughout 5 minutes of light exposure, and the content of the free thiol was immediately assayed with Ellman's reagent buffer. The thiol concentration presented at the specific time was measured against a known standard (i.e., L-cysteine $\mathrm{HCl}$ ). For norbornene conversion, non-gelling precursor solutions were exposed to visible light for 5 minutes, followed by dialysis against ddH2O to remove small molecular weight by-products. The samples were freeze-dried, dissolved in chloroform-d, and subjected to proton NMR evaluation. ${ }^{1} \mathrm{H}$ NMR was used to evaluate the signature peaks for ethyl 
protons on PEG backbone ( $\delta$ 3.9-4.2 ppm, peaks a) and norbornene ( $\delta$ 5.8-6.2 ppm, peaks b, c). The areas of the norbornene peaks were obtained and integrated areas obtained were further compared to the area under the norbornene peaks for pure PEGdNB at the same macromer concentration. Norbornene consumption was calculated using the difference in the area of the norbornene peaks before and after reaction dived by the area before the reaction.

\section{UVIVis spectrophotometry}

Eosin-Y (at $0.1 \mathrm{mM}$ ) and various components (i.e., PEGdNB $6 \mathrm{kDa}$, L-cysteine $\mathrm{HCl}$, and Ltyrosine methyl ester) were dissolved in PBS ( $\mathrm{pH}$ 7.4) and exposed to visible light for 5 minutes. Non-gelling components were used (e.g., PEGdNB and L-cysteine) to prevent gelation and to facilitate solution-based UV/Vis spectrometric measurements. Concentrations of the components were equivalent to those used in gelation studies (final concentration of norbornene and thiol $=$ $12 \mathrm{mM}$ ). Using a microplate reader (BioTek Synergy HT) in UV/Vis absorption mode (wavelength between 400 and $700 \mathrm{~nm}$ at $2 \mathrm{~nm}$ increments), the absorbance spectra of the non-gelling solutions were measured before and after the exposure of light. To prevent saturation in absorbance, solution was diluted so the final concentration of eosin-Y was $0.02 \mathrm{mM}$.

\section{Cell encapsulation and viability}

Mouse insulinoma cells (MIN6, a gift from Prof. Raghu Mirmira of Indiana University School of Medicine), were suspended in polymer solutions containing $0.1 \mathrm{mM}$ eosin-Y, PEG8aNB, and di-cysteine peptide (i.e., Lys-Cys-Tyr-lle-Gly-Ser-Arg-Cys-Lys or KCYIGSRCK) with and without L-tyrosine methyl ester. Precursor solution was exposed to the same visible light for 5 minutes to obtain cell-laden hydrogels (cell density in the gels at $5 \times 10^{6}$ cells $/ \mathrm{mL}, 20 \mu \mathrm{l} / \mathrm{gel}$ ), which were maintained in high-glucose DMEM supplemented with $10 \%$ fetal bovine serum (FBS), 50 $\mu \mathrm{M} \beta$-mercaptoethanol, and $1 \times$ antibiotic-antimycotic. To characterize cell viability, cell-laden hydrogels were incubated in $500 \mu \mathrm{L}$ Almarblue ${ }^{\circledR}$ reagent $\left(10 \%\right.$ in cell culture medium) at $37^{\circ} \mathrm{C}$ and $5 \%$ of $\mathrm{CO}_{2}$. After 16 hours of incubation, $200 \mu \mathrm{l}$ of media were transferred to a 96 -well plate 
for fluorescence quantification (excitation: $560 \mathrm{~nm}$ and emission: $590 \mathrm{~nm}$ ). To obtain qualitative cell viability, cells were stained with a live/dead staining kit (Calcein AM stained live cells green and Edithium homodimer stained dead cells red) and imaged with confocal microscopy (Olympus FV-1000 Laser Scanning Biological Microscope). For MIN6 encapsulated gels, three images were taken at a step size of $10 \mu \mathrm{m}$ for a total depth of $100 \mu \mathrm{m}$ per image.

\section{Insulin secretion from $\beta$-cells}

To access glucose stimulated insulin secretion (GSIS), cell-laden hydrogels were rinsed with HBSS, followed by priming in Krebs-Ringer bicarbonate (KRB) buffer (23 mM sodium chloride, $1 \mathrm{mM}$ potassium chloride, $4.8 \mathrm{mM}$ sodium bicarbonate, $0.2 \mathrm{mM}$ magnesium chloride hexahydrate, $0.2 \mathrm{mM}$ calcium chloride dehydrate, $0.5 \mathrm{mM}$ 4-(2-hydroxyethyl)-1-piperazineethanesulfonic acid and 0.1 vol\% bovine serum albumin) containing $2.5 \mathrm{mM}$ glucose for 1 hour at $37^{\circ} \mathrm{C}$ and $5 \% \mathrm{CO}_{2}$. Then, the gels were incubated with $500 \mu \mathrm{L}$ of low $(2.5 \mathrm{mM})$ or high $(25 \mathrm{mM})$ glucose KRB for 2 hours each. The low and high glucose buffer solutions were collected and analyzed using mouse

insulin ELISA kit (Mercodia). Cell viability was further quantified by CellTiter Glo® assay (Promega). Insulin secretion obtained from ELISA was normalized by the respective ATP content of the cell-laden hydrogels.

\section{Data analysis}

All experiments were conducted independently for three times and results were reported as mean \pm SD. Data was analyzed with student's t-test using GraphPad Prism $5(p<0.05)$.

\section{RESULTS AND DISCUSSION}

Tyrosine-assisted crosslinking of thiol-norbornene PEG-peptide hydrogels

We first evaluate the influence of soluble tyrosine on the gelation kinetics of PEG-peptide hydrogels formed by visible light initiated thiol-norbornene polymerization. Gelation was carried 
out using PEG8NB, a bis-cysteine-containing peptide crosslinker (i.e., CGGGC), and photoinitiator eosin-Y (Figure 1A). Soluble tyrosine was also added in some samples. The gelation kinetics was monitored by in situ photorheometry using a visible light source (Figure 1B and 1C). We found that PEG-peptide hydrogels crosslinked without soluble tyrosine had a gel point of about 100 seconds. After 300 seconds of visible light exposure, the shear modulus was only about $100 \mathrm{~Pa}$ (Figure 1B), indicative of poor gelation efficiency as compared with the gelation using 4-arm PEGNB and an inert crosslinker DTT [48]. Since visible light-mediated thiolnorbornene gelation starts with the deprotonation of thiol group by excited eosin-Y, we suspected that the poor gelation efficiency shown in Figure 1B might be a result of insufficient deprotonation of thiol groups on cysteine residues, which were part of the peptide sequence. Furthermore, we found that gelation efficiency depends highly on the sequence of the bis-cysteine peptide crosslinkers (Figure S1). It has been reported that some amino acids (e.g., tyrosine, tryptophan) could increase the half-life of eosin- $Y$ radicals $[49,50]$. Therefore, we hypothesized that the addition of soluble tyrosine might enhance the crosslinking efficiency of visible light-based thiolnorbornene hydrogels through improving eosin-Y mediated cysteine deprotonation. To test this hypothesis, $1 \mathrm{mM}$ of soluble tyrosine was added in the prepolymer solution. The addition of soluble tyrosine shortened the gel point by five-fold to $\sim 22$ seconds, and increased the shear modulus of the gel by ten-fold to $\sim 1,000 \mathrm{~Pa}$ (Figure 1C). The significant improvement of gelation efficiency in the presence of soluble tyrosine was indicative of an accelerated deprotonation of peptide-bound cysteine side-chain, which enhanced the thiol-norbornene coupling.

\section{Conversion of functional groups}

To examine the kinetics of tyrosine-assisted thiol-norbornene reaction, we characterized thiol and norbornene consumptions in the presence of soluble tyrosine at different concentrations. In order to facilitate quantification of the thiol/norbornene consumptions, macromer components were selected such that no crosslinked network was formed following visible light exposure (i.e., 
linear PEG-norbornene or PEGdNB, and soluble cysteine). Eosin-Y was used at $0.1 \mathrm{mM}$ and soluble tyrosine was added at different concentrations (i.e., 0, 0.1, 1, $10 \mathrm{mM}$ ). After visible light exposure, the concentrations of free thiol remaining in the solution were quantified by Ellman's assay and converted to percent thiol conversion. Figure $\mathbf{2}$ shows that percent thiol conversion was higher when soluble tyrosine was added at higher concentrations. For example, thiol conversion at 20 seconds (where gel point was previously determined, Figure 1C) increased from $-22 \%$ to $\sim 48 \%$ as the concentration of soluble tyrosine was increased from 0 to $10 \mathrm{mM}$ (Figure 2, right panel). The higher thiol conversion in the presence of soluble tyrosine could explain the faster gel point shown in Figure 1C. Interestingly, while the in situ photorheometry results showed that final gel moduli were affected by the presence of soluble tyrosine (Figure 1B and 1C), all non-gelling formulations eventually reached similar thiol conversion regardless of the amount of soluble tyrosine added (Figure 2, $\sim 95 \%$ at 300 seconds of visible light exposure). We believe that this was not caused by solution acidity change due to the addition of tyrosine as we found no significant differences in $\mathrm{pH}$ values of the non-gelling solution containing different tyrosine concentrations, either with or without cysteine (Figure S2). It was possible that the thiolnorbornene reaction efficiency was higher in the non-gelling experiment than in the actual network crosslinking owing to the fact that some reactions in the latter led to dangling chains (i.e., network defects) that did not contribute to shear moduli of the resulting hydrogels. It was also likely that some deprotonated thiols formed disulfide bonds that did not contribute to network crosslinking. Nonetheless, the presence of soluble tyrosine increased the efficiency of thiol deprotonation, which was an important factor in the thiol-norbornene gelation.

In addition to thiol conversion, we examined the consumption of norbornene groups in similar visible light initiated non-gelling thiol-norbornene reactions. Following the non-gelling reactions, the samples were dialyzed against $\mathrm{ddH}_{2} \mathrm{O}$ to remove small molecular weight byproducts (e.g., photoinitiator fragments, tyrosine, cysteine, etc.). The freeze-dried samples were 
dissolved in chloroform-d and subjected to proton NMR evaluation. The areas under the norbornene peaks (Figure 3A, $\delta 5.8-6.2 \mathrm{ppm}$ ) were integrated to obtain the norbornene conversion. As the concentration of soluble tyrosine was increased from 0 to $10 \mathrm{mM}$, the areas under the norbornene peaks decreased from 0.753 to 0.454 a.u. (Figure 3B). Correspondingly, norbornene consumption increased from $\sim 19 \%$ to $51 \%$ when the concentration of soluble tyrosine was increased from 0 to $10 \mathrm{mM}$ (Figure 3B). Since the step-growth thiol-norbornene reaction is strictly orthogonal (i.e., no homo-polymerization between norbornene groups under current experimental conditions), the amount of norbornene consumed after the reaction could be attributed to thiol-norbornene coupling. It is worth noting that the degree of norbornene conversion was not as high as the thiol conversion even though all reaction mixtures were composed of equal molarity of thiol and norbornene groups. It was possible that, upon visible light exposure the excited eosin-Y abstracted protons from cysteinyl thiols and promoted other undesired thiol-based reactions (in addition to the thiol-norbornene reaction) including disulfide bond formation or other reactions that depleted free thiols. It was worth noting that, even in the presence of high tyrosine concentration (i.e., 10mM), only about half of the available norbornene moieties were consumed in the 'non-gelling' experiment, suggesting that other factors exist to complicate the visible light mediated thiol-norbornene reactions. While functional group conversion (either thiol or norbornene) in a 'non-gelling' experiment is not equivalent quantitatively to that happens during actual network crosslinking, the results from these 'non-gelling' experiments nonetheless have demonstrated that the addition of soluble tyrosine indeed improved the conjugation efficiency between cysteinyl thiol and norbornene groups. Since it is not unusual to observe network nonideality in step-growth hydrogels (especially with lower macromer concentrations) [31, 52], the enhanced visible light based thiol-norbornene reaction efficiency in the presence of soluble tyrosine provides an easy and effective route of improving the crosslinking visible light based thiolnorbornene hydrogels. 


\section{Effect of tyrosine, macromer content and thiol-to-norbornene ratio on hydrogel properties}

As stated above, the addition of soluble tyrosine could improve thiol-norbornene reaction efficiency. Here, we asked whether adding soluble tyrosine would improve the properties of visible light based thiol-norbornene PEG-peptide hydrogels. We utilized a simple bis-cysteine containing peptide (sequence: CGGGC) as the crosslinker and added soluble tyrosine at different concentrations in the pre-polymer solutions. PEG8NB-CGGGC hydrogels were prepared using the same visible light and eosin-Y mediated thiol-norbornene crosslinking and the hydrogels were characterized through measuring gel fraction, elastic modulus, and swelling ratio. As the concentration of soluble tyrosine was increased from 0 to $1 \mathrm{mM}$, gel fraction increased from 50 to $65 \%$ (Figure 4A), elastic modulus increased from 1 to $3 \mathrm{kPa}$ (Figure 4B), and equilibrium swelling ratio decreased from $\sim 16$ to $\sim 10$ (Figure $4 \mathrm{C}$ ). These metrics (i.e., accelerated gel points, increased gel modulus, improved functional group conversion, and changes in gel swelling ratio) were indicative of an improved crosslinking in the presence of soluble tyrosine. Interestingly, further increasing tyrosine from $1 \mathrm{mM}$ to $10 \mathrm{mM}$ led to a slight decrease in gel elastic modulus, as well as an increased in swelling ratio. The negative impact of high tyrosine concentration on gel properties was unexpected as the results obtained from thiol and norbornene consumption (Figures 2 and $\mathbf{3}$ ) showed a monotonic increase of functional group conversion. It is worth noting that higher functional group conversion seen in the non-gelling experiments could not be directly correlated to improved network crosslinking owing to the presence of network non-ideality. The presence of high content soluble tyrosine could also lead to undesired reactions, which complicate the experimental results. As in the other chemically crosslinked thiol-norbornene hydrogels, the physical properties of gels could still be tuned by varying PEG8NB macromer and the ratio of norbornene to thiol. In the presence of $1 \mathrm{mM}$ soluble tyrosine during gelation, elastic moduli of visible light crosslinked thiol-norbornene hydrogels increased 25-fold (i.e., from $1.3 \mathrm{kPa}$ to $32 \mathrm{kPa}$ ) when PEG8NB was increased from $3 \mathrm{wt} \%$ to $9 \mathrm{wt} \%$ (Figure S3A). Also in the presence of $1 \mathrm{mM}$ soluble tyrosine, increasing ratio of norbornene to thiol from 0.6 to 1 led to a 26 -fold increase in 
elastic moduli (Figure S3B, 0.05 to $1.3 \mathrm{kPa}$ ). These studies demonstrated the high tunability of visible light polymerized step-growth PEG-peptide hydrogels [48, 52].

\section{Effect of soluble tyrosine on the spectrophotometric properties of eosin-Y}

In addition to the alterations in gelation kinetics and hydrogel properties, we observed changes in physical appearance of the hydrogels in the presence of soluble tyrosine during visible light initiated thiol-norbornene gelation. The visible light crosslinked thiol-norbornene hydrogels were normally pink to red in color due to the presence of eosin-Y [53]. However, the addition of soluble tyrosine appeared to quench the redness of hydrogels in a dose-dependent manner (Figure 5A). Since the concentration of eosin-Y was the same across the four formulations, the disappearance of gel redness was potentially due to the quenching of eosin-Y by tyrosine through a proton-coupled electron transfer process $[54,55]$. To verify this, we evaluated the spectrophotometric properties of precursor solution containing non-gelling components. Before exposing solution to light, the UV/Vis spectra of these solution were similar regardless of the concentration of tyrosine used (peak absorbance of $\sim 0.5$ at $516 \mathrm{~nm}$, Figure 5B to 5E). However, after exposing the non-gelling solution to visible light, the peak absorbance (at $516 \mathrm{~nm}$ ) of the solution decreased with increasing concentration of tyrosine (Figure 5B to 5E, 0.25 to 0.18 a.u. for $0 \mathrm{mM}$ to $10 \mathrm{mM}$ of tyrosine, respectively), which suggest that the presence of soluble tyrosine quenched the red color of eosin-Y. We believe that the presence of soluble tyrosine during visible light based thiol-norbornene photocrosslinking caused changes in eosin-Y excitation status, which led to enhanced deprotonation of thiol groups and increased thiol-norbornene reaction and gel crosslinking. The reduction in red color has an added benefit in fluorescence imaging as there was some overlapping in the emission spectra of eosin-Y and DNA labeling dyes (e.g., edithium homodimer 1). The quenching of eosin-Y by soluble tyrosine following photo-crosslinking is beneficial as this reduces the background fluorescence attributed by eosin-Y. 


\section{Cytocompatibility of tyrosine-assisted visible light-based thiol-norbornene PEG-peptide gel crosslinking}

To explore the potential of tyrosine-assisted, visible light-based thiol-norbornene PEGpeptide gel crosslinking in tissue engineering applications, we evaluated the cytocompatibility of this gelatin system using in situ encapsulation of pancreatic MIN6 $\beta$-cells. In this experiment, we utilized non-degradable 8-arm PEGNB (PEG8aNB, $20 \mathrm{kDa}$ ) as the base macromer to afford hydrolytically stable hydrogels throughout the course of study (Figure S4). In addition, we synthesized a laminin-derived peptide flanking with terminal cysteine residues (i.e., KCYIGSRCK, terminal lysine residues added to increase peptide solubility) as the hydrogel crosslinker to provide biomimicry to the otherwise inert PEG-based hydrogels [56-58]. Since the addition of soluble tyrosine during thiol-norbornene photopolymerization increased stiffness of the resulting gels, which could be a confounding factor when evaluating cellular fate, we formulated the cellladen hydrogels such that the elastic moduli of all hydrogels were similar (Figure S4, $\sim 2 \mathrm{kPa}$ ). This was achieved through adjusting down the stoichiometric ratio of thiol to norbornene when 1 $\mathrm{mM}$ of soluble tyrosine was added to the prepolymer solution (i.e., $\mathrm{R}_{\text {[thiol][ene] }}=1$ for gelation without tyrosine and 0.85 for gelation with $1 \mathrm{mM}$ of tyrosine).

After cell encapsulation, live/dead staining was performed and more than $80 \%$ of the MIN6 $\beta$-cells were stained green, indicating that the gelation was cytocompatible for in situ cell encapsulation. However, we observed slightly more dead cells $(\sim 10 \%)$ when encapsulation was performed without soluble tyrosine (Figure 6A, top). Regardless of the initial cell viability, $\beta$-cells formed cell spheroids after 10 days of culture in both formulations (Figure 6A, bottom). In addition, AlamarBlue ${ }^{\circledR}$ reagent was used to quantify metabolic activity of the cells encapsulated within these hydrogels. Compared with cells encapsulated in the absence of tyrosine, cells encapsulated in the presence of tyrosine showed higher cell metabolic activity throughout the course of study (Figure 6B). Furthermore, $\beta$-cells encapsulated in the presence of tyrosine had higher insulin 
secretion than cells encapsulated without the addition of tyrosine (Figure 6C, 2-fold and 3-fold higher on day 1 and 10, respectively). We further examined the expression of mouse insulin (ins) and pancreatic duodenal homeobox-1 $(p d x 1)$ on the mRNA level. These two genes were chosen because of their importance in glycemic control and in proliferation of pancreatic $\beta$-cells $[59,60]$. The mRNA expression of ins and $p d x 1$ showed that the addition of tyrosine did not create adverse influence on $\beta$-cells (Figure S5). In addition to encapsulating MIN6 $\beta$-cells, we also performed in situ encapsulation using isolated mouse islets (from CD1 mice). Live/dead staining images of the encapsulated islets showed that more dead cells when islets were encapsulated in the absence of tyrosine (Figure S6). These results confirmed that the addition of tyrosine during gelation did not negatively impact $\beta$-cells and islets viability.

Previous studies have shown that insulin secretion from islets and $\beta$-cells could be upregulated by selected amino acids [61]. To evaluate if the viability of encapsulated $\beta$-cells was influenced by the presence of soluble tyrosine, we performed a control study where cell encapsulation was performed in the absence of tyrosine. Following encapsulation, cell-laden gels were placed in media in the absence or presence of tyrosine (at $1 \mathrm{mM}$ ) for one hour prior to analyses. An incubation period of one hour was selected as it was sufficiently for tyrosine to diffuse into the cell-laden hydrogels. The live/dead staining images and Alarmarblue ${ }^{\circledR}$ reagent assay results showed that $\beta$-cells had similar viability and metabolic activity regardless of the media condition (i.e., with or without soluble tyrosine, Figure S7). These results indicate that the presence of soluble tyrosine alone was not sufficient to influence cell viability. Taken together, the results in Figure 6 and Figure S7 suggest that the presence of soluble tyrosine during visible light based thiol-norbornene gelation likely attenuated some adverse effects of excited eosin-Y radicals on cell viability. Although the current work demonstrates that the gelation efficiency and cytocompatibility of visible light based thiol-norbornene PEG-peptide hydrogels can be significantly improved by adding soluble tyrosine during gel crosslinking, future mechanistic work 
is necessary to elucidate the potential biological effects of soluble tyrosine on $\beta$-cells viability and function.

\section{CONCLUSIONS}

In summary, we have synthesized visible light mediated step-growth thiol-norbornene photopolymerized hydrogel using multi-arm PEGNB and di-cysteine containing peptides. We found that thiol-norbornene hydrogels crosslinked in the presence of soluble tyrosine exhibited faster gelation kinetics and higher crosslinking efficiency. Furthermore, the network crosslinking depended highly on the amount of tyrosine, concentration of macromer, and the ratio of norbornene to thiol used. In addition, a series of cell viability tests revealed that hydrogels crosslinked in the presence of tyrosine preserved the viability and insulin secreting function of pancreatic $\beta$-cells. Together, these data suggest that tyrosine-assisted visible light mediated thiolnorbornene polymerization should be of great value for fabricating PEG-peptide hydrogels in tissue engineering applications.

\section{ACKNOWLEDGEMENTS}

This project was funded in part by the National Science Foundation CAREER award (DMR \#1452390), a Pilot Funding for Research Use of Core Facilities from Indiana Clinical \& Translational Sciences Institute, and Purdue Research Foundation PhD Fellowship (to HS).

\section{SUPPORTING INFORMATION}

Supporting methods for islet isolation and characterization, as well as for mRNA isolation of real time PCR. Supporting figures for additional material characterizations and islet encapsulation results. 


\section{FIGURE CAPTIONS}

Figure 1. (A) Schematic of visible light and eosin-Y mediated thiol-norbornene gelation. (B) In situ photorheometry of thiol-norbornene photopolymerization between PEG8NB (3 wt\%) and CGGGC $(6 \mathrm{mM})$ in the absence of soluble tyrosine. (C) Gelation in the presence of $1 \mathrm{mM}$ soluble tyrosine. G': storage modulus; G": loss modulus. Eosin-Y: 0.1 mM. Light intensity: 70 kLux. n= 3. Error bars were omitted for clarity.

Figure 2. Thiol conversion quantified by Ellman's assay. Non-gelling components used in the reaction mixtures were: 3.6 wt\% PEGdNB (i.e., 12 mM norbornene), 12 mM L-cysteine (i.e., 12 $\mathrm{mM}$ thiol), $0.1 \mathrm{mM}$ eosin-Y and different concentrations of soluble tyrosine $(n=3)$. The right panel shows the shaded region in the left plot.

Figure 3. (A) Effect of soluble tyrosine on norbornene conversion as determined by ${ }^{1} \mathrm{H}$ NMR using non-gelling components: PEGdNB (3.6 wt\%), soluble cysteine (12 mM), eosin-Y (0.1 mM), and tyrosine $(0,0.1,1$, and $10 \mathrm{mM})$. All samples were prepared in $\mathrm{D}_{2} \mathrm{O}$ (at $\left.4.8 \mathrm{ppm}\right)$. (B) Integration of the areas under the norbornene peaks and calculated norbornene group conversion in the presence of different soluble tyrosine concentration.

Figure 4. Effect of soluble tyrosine on (A) gel fraction, (B) elastic moduli, and (C) swelling ratio. Gelation was performed with 3 wt $\%$ PEG8NB, 6 mM CGGGC, $0.1 \mathrm{mM}$ eosin-Y, and different concentrations of soluble tyrosine under visible light exposure. ${ }^{*}$ denotes $p<0.05$.

Figure 5. (A) Photograph of hydrogels at equilibrium swelling (3wt\% PEG8NB, and 6 mM CGGGC). (B-E) UV-vis spectra of eosin-Y before (thick blue) and after (thin red) visible light exposure for 5 minutes using non-gelling components: 3.6 wt\% PEGdNB; 6 mM CGGGC, and 
different concentrations of soluble tyrosine: (B) $0 \mathrm{mM},(C) 0.1 \mathrm{mM}$, (D) $1 \mathrm{mM}$, and (E) $10 \mathrm{mM}$. Eosin-Y concentration in all measurements was $0.1 \mathrm{mM}$.

Figure 6. Effect of soluble tyrosine on the cytocompatibility of MIN6 $\beta$-cells following visible light initiated thiol-norbornene gelation. (A) Representative confocal z-stack images of MIN6 cells stained with live/dead staining kit on day 1 (Scales: $100 \mu \mathrm{m})$. (B) Cell viability as assessed by Alamarblue ${ }^{\circledR}$ reagent. (C) Glucose stimulated insulin secretion ( $25 \mathrm{mM}$ of glucose) normalized by cell metabolic activity. * indicates comparison between day 1 and day 10 , while \% indicates comparison between 0 to $1 \mathrm{mM}$ of tyrosine, $p<0.05$. All gel formulations contained $\beta$-cells at $5 \times 10^{6}$ cells/mL, 3.5 wt\% PEG8aNB, KCYIGSRCK, $0.1 \mathrm{mM}$ eosin-Y. Ratio of [thiol] to [norbornene] was 1 and 0.85 for $0 \mathrm{mM}$ and $1 \mathrm{mM}$ of tyrosine, respectively 
Figure 1

A.
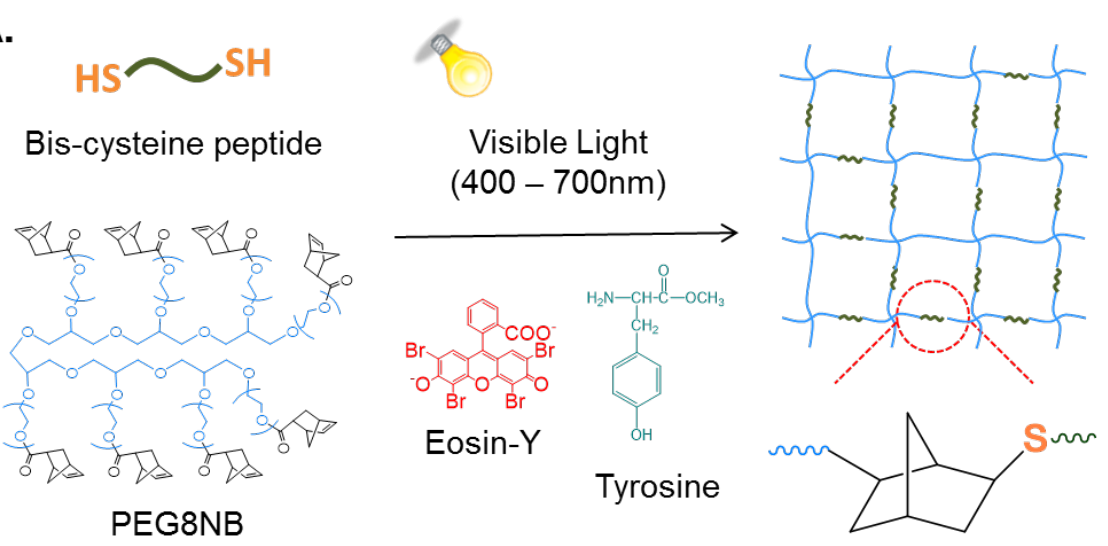

B.

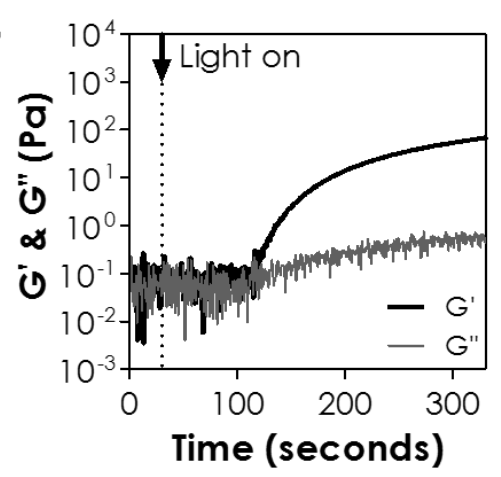

c.

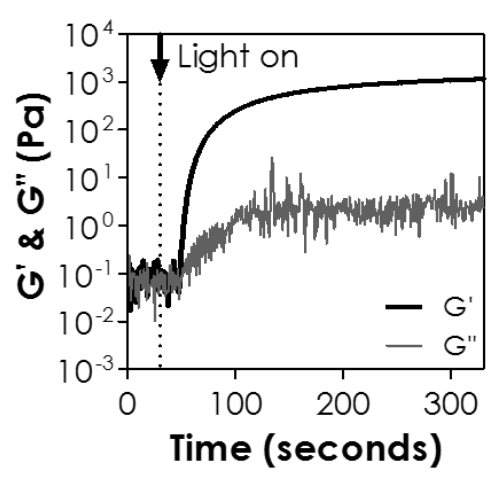


Figure 2
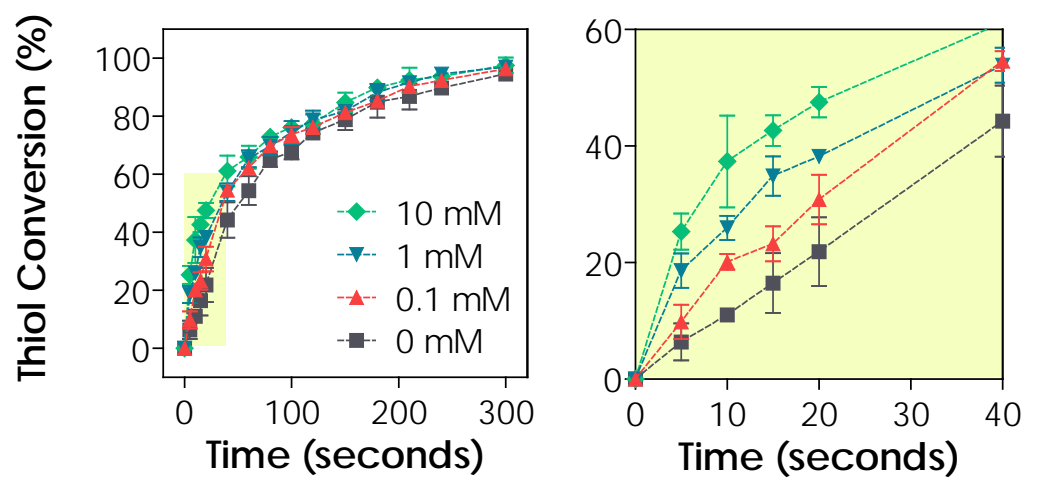
Figure 3

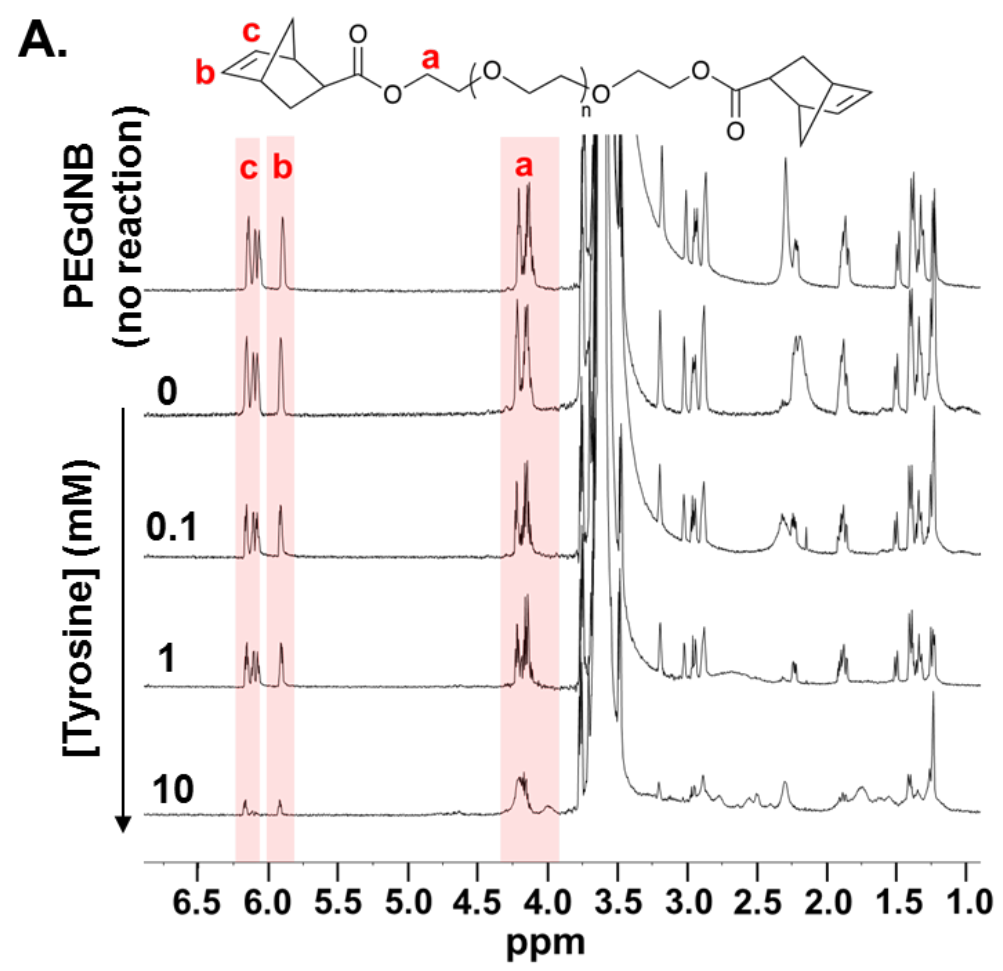

\begin{tabular}{|l|c|c|c|}
\cline { 2 - 4 } \multicolumn{1}{c|}{} & $\begin{array}{c}\text { [Tyrosine] } \\
(\mathbf{m M})\end{array}$ & $\begin{array}{c}\mathrm{A}_{\mathrm{b}+\mathrm{c}} \\
\text { (a.u.) }\end{array}$ & $\begin{array}{c}\text { Norbornene } \\
\text { conversion (\%) }\end{array}$ \\
\hline \multirow{4}{*}{ (-)reaction } & 0 & 0.929 & 0 \\
\hline \multirow{3}{*}{ (+)reaction } & 0 & 0.753 & 18.96 \\
\cline { 2 - 4 } & 0.1 & 0.644 & 30.67 \\
\cline { 2 - 4 } & 1 & 0.520 & 44.09 \\
\cline { 2 - 4 } & 10 & 0.454 & 51.19 \\
\hline
\end{tabular}


Figure 4

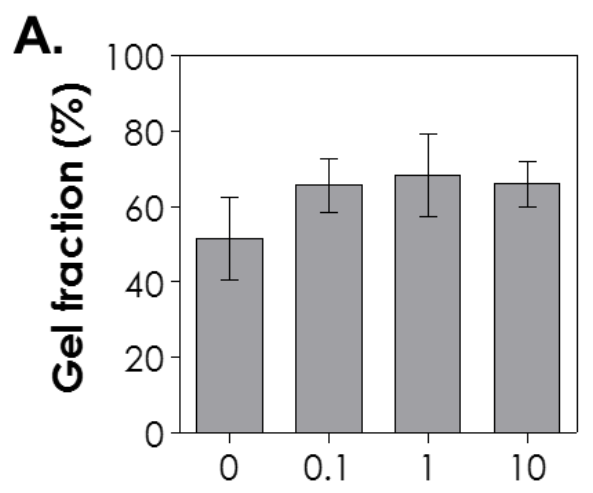

[Tyrosine] (mM)

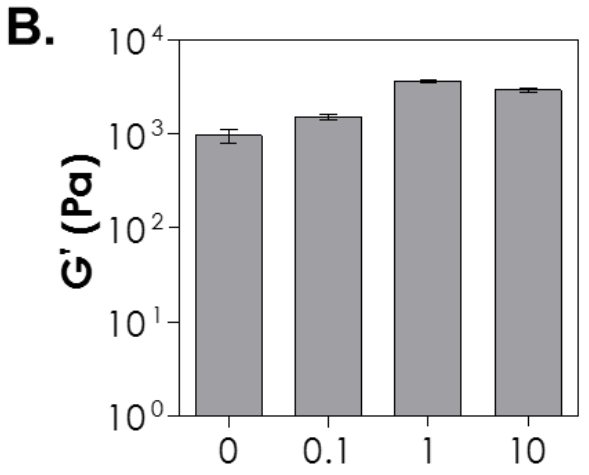

[Tyrosine] (mM)

C.

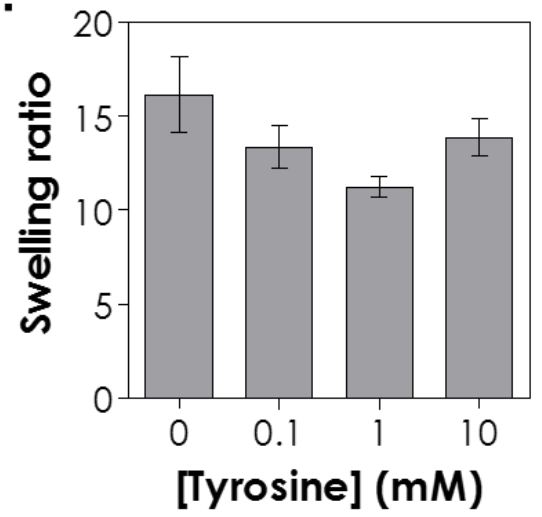


Figure 5

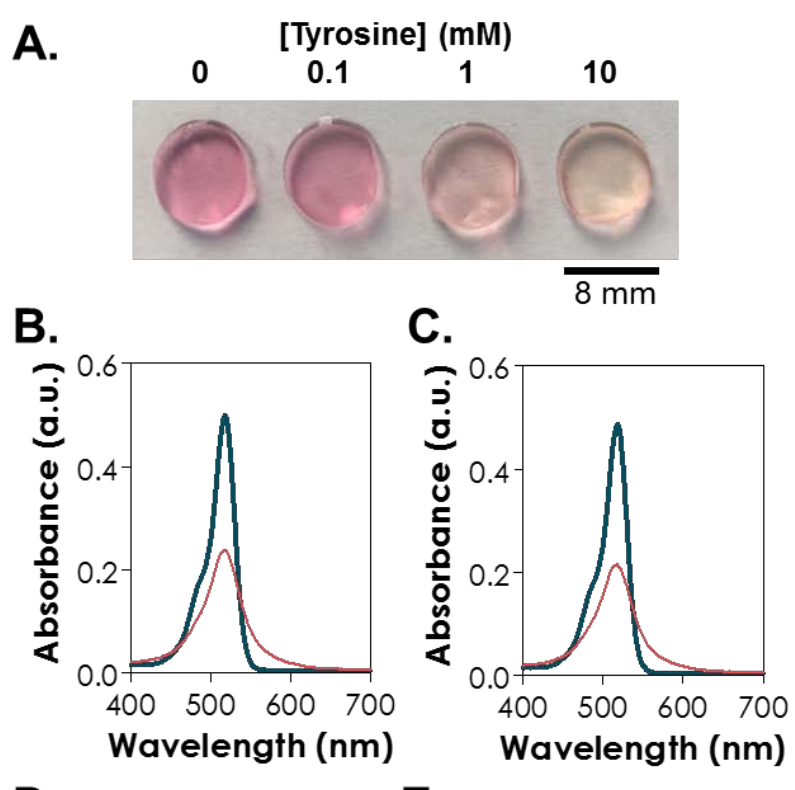

D.

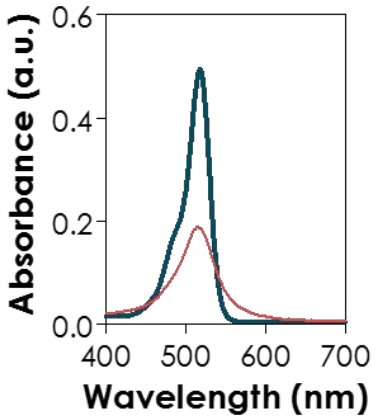

E.

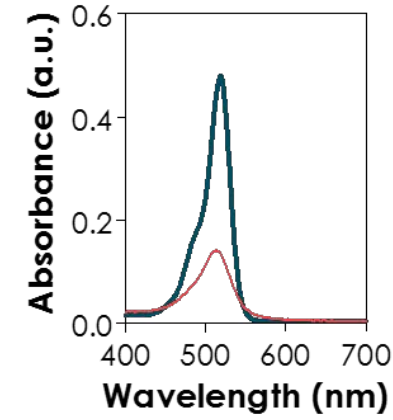


Figure 6
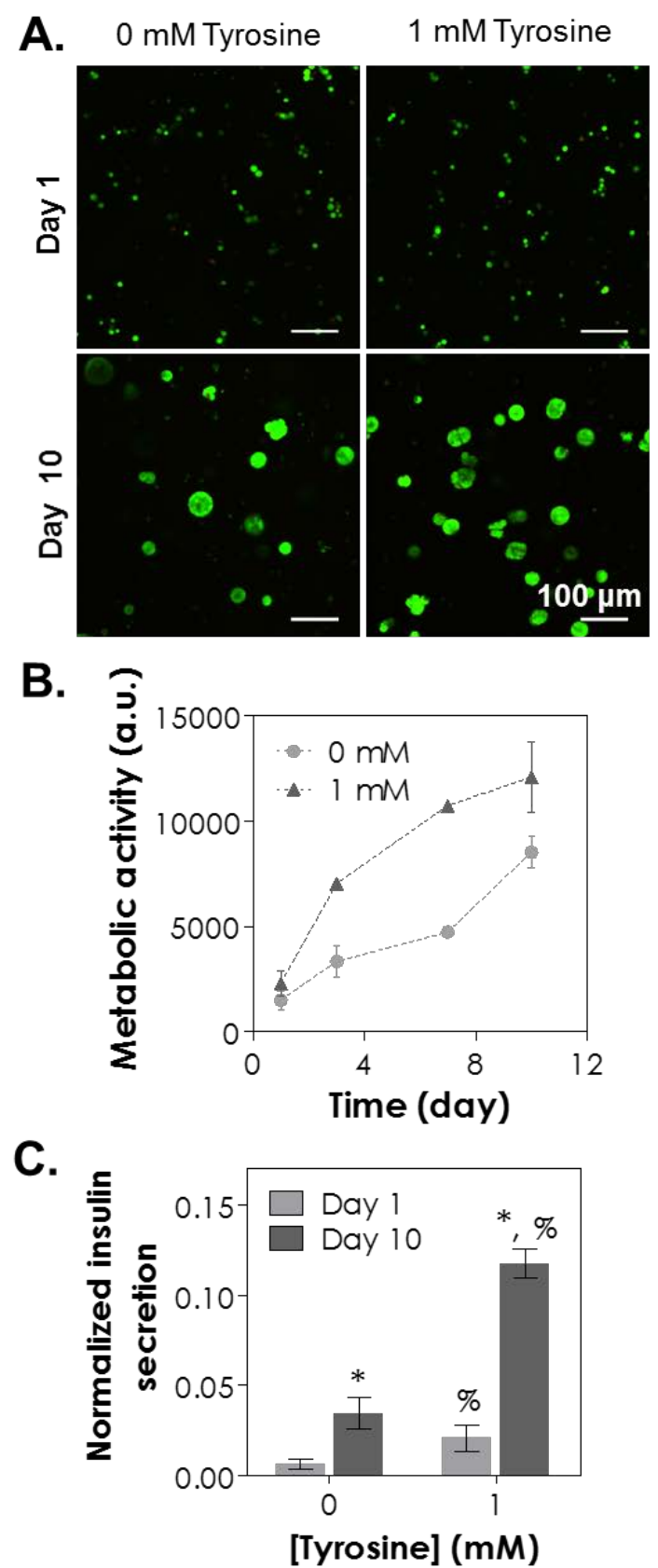


\section{REFERNCES}

[1] Simmons CA, Alsberg E, Hsiong S, Kim WJ, Mooney DJ. Dual growth factor delivery and controlled scaffold degradation enhance in vivo bone formation by transplanted bone marrow stromal cells. Bone 2004;35:562-9.

[2] Zhao Y, Nakajima T, Yang JJ, Kurokawa T, Liu J, Lu J, et al. Proteoglycans and glycosaminoglycans improve toughness of biocompatible double network hydrogels. Adv Mater 2014;26:436-42.

[3] Chen WY, Abatangelo G. Functions of hyaluronan in wound repair. Wound Repair Regen 1999;7:79-89.

[4] Choi WI, Kim M, Tae G, Kim YH. Sustained release of human growth hormone from heparinbased hydrogel. Biomacromolecules 2008;9:1698-704.

[5] Dudas J, Ramadori G, Knittel T, Neubauer K, Raddatz D, Egedy K, et al. Effect of heparin and liver heparan sulphate on interaction of HepG2-derived transcription factors and their cisacting elements: altered potential of hepatocellular carcinoma heparan sulphate. Biochem J 2000;350 Pt 1:245-51.

[6] Homminga GN, Buma P, Koot HWJ, van der Kraan PM, van den Berg WB. Chondrocyte behavior in fibrin glue in vitro. Acta Orthop 1993;64:441-5.

[7] Thompson DF, Letassy NA, Thompson GD. Fibrin glue: a review of its preparation, efficacy, and adverse effects as a topical hemostat. Drug Intell Clin Pharm 1988;22:946-52.

[8] Murata $\mathrm{Y}$, Kontani $\mathrm{Y}$, Ohmae $\mathrm{H}$, Kawashima S. Behavior of alginate gel beads containing chitosan salt prepared with water-soluble vitamins. Eur J Pharm Biopharm 2002;53:249-51.

[9] Lin Y-H, Liang H-F, Chung C-K, Chen M-C, Sung H-W. Physically crosslinked alginate/N,Ocarboxymethyl chitosan hydrogels with calcium for oral delivery of protein drugs. Biomaterials 2005;26:2105-13.

[10] Lee KW, Yoon JJ, Lee JH, Kim SY, Jung HJ, Kim SJ, et al. Sustained release of vascular endothelial growth factor from calcium-induced alginate hydrogels reinforced by heparin and chitosan. Transplant Proc 2004;36:2464-5.

[11] Hennink WE, De Jong SJ, Bos GW, Veldhuis TF, van Nostrum CF. Biodegradable dextran hydrogels crosslinked by stereocomplex formation for the controlled release of pharmaceutical proteins. Int J Pharm 2004;277:99-104.

[12] Mao C, Kisaalita WS. Characterization of 3-D collagen hydrogels for functional cell-based biosensing. Biosens Bioelectron 2004;19:1075-88.

[13] Tabata Y, Hijikata S, Muniruzzaman M, Ikada Y. Neovascularization effect of biodegradable gelatin microspheres incorporating basic fibroblast growth factor. J Biomater Sci Polym Ed 1999;10:79-94.

[14] Yamamoto $\mathrm{M}$, Takahashi $\mathrm{Y}$, Tabata $\mathrm{Y}$. Controlled release by biodegradable hydrogels enhances the ectopic bone formation of bone morphogenetic protein. Biomaterials 2003;24:4375-83.

[15] Guvendiren M, Burdick JA. Engineering synthetic hydrogel microenvironments to instruct stem cells. Curr Opin Biotechnol 2013;24:841-6.

[16] Brinkman E, van der Does L, Bantjes A. Poly(vinyl alcohol)-heparin hydrogels as sensor catheter membranes. Biomaterials 1991;12:63-70. 
[17] Peppas NA, Scott JE. Controlled release from poly ( vinyl alcohol ) gels prepared by freezingthawing processes. J Control Release 1992;18:95-100.

[18] Stauffer SR, Peppast NA. Poly(vinyl alcohol) hydrogels prepared by freezing-thawing cyclic processing. Polymer 1992;33:3932-6.

[19] Hern DL, Hubbell JA. Incorporation of adhesion peptides into nonadhesive hydrogels useful for tissue resurfacing. J Biomed Mater Res 1998;39:266-76.

[20] Salinas CN, Anseth KS. Mixed Mode Thiol-Acrylate Photopolymerizations for the Synthesis of PEG-Peptide Hydrogels. Macromolecules 2008;41:6019-26.

[21] Lin C-C, Anseth KS. Glucagon-Like Peptide-1 Functionalized PEG hydrogels promote survival and function of encapsulated pancreatic $\beta$-cells. Biomacromolecules 2009;10:24607.

[22] Elbert DL, Pratt AB, Lutolf MP, Halstenberg S, Hubbell JA. Protein delivery from materials formed by self-selective conjugate addition reactions. J Control Release 2001;76:11-25.

[23] Lutolf MP, Lauer-Fields JL, Schmoekel HG, Metters AT, Weber FE, Fields GB, et al. Synthetic matrix metalloproteinase-sensitive hydrogels for the conduction of tissue regeneration: Engineering cell-invasion characteristics. Proc Natl Acad Sci U S A 2003;100:5413-8.

[24] Zustiak SP, Leach JB. Hydrolytically degradable poly(ethylene glycol) hydrogel scaffolds with tunable degradation and mechanical properties. Biomacromolecules 2010;11:1348-57.

[25] Zustiak SP, Leach JB. Characterization of Protein Release From Hydrolytically degradable poly(ethylene glycol) hydrogels. Biotechnol Bioeng 2011;108:197-206.

[26] Phelps EA, Templeman KL, Thulé PM, García AJ. Engineered VEGF-releasing PEG-MAL hydrogel for pancreatic islet vascularization. Drug Deliv Transl Res 2013;5:125-36.

[27] Metters AT, Anseth KS, Bowman CN. Fundamental studies of a novel, biodegradable PEGb-PLA hydrogel. Polymer 2000;41:3993-4004.

[28] Metters AT, Bowman CN, Anseth KS. A statistical kinetic model for the bulk degradation of PLA-b-PEG-b-PLA hydrogel networks. J Phys Chem B 2000;104:7043-9.

[29] Metters AT, Anseth KS, Bowman CN. A statistical kinetic model for the bulk degradation of PLA-b-PEG-b-PLA hydrogel networks: Incorporating network non-idealities. J Phys Chem B 2001;105:8069-76.

[30] Lin-Gibson S, Jones RL, Washburn NR, Horkay F. Structure-property relationships of photopolymerizable poly(ethylene glycol) dimethacrylate hydrogels. Macromolecules 2005;38:2897-902.

[31] Metters A, Hubbell J. Network formation and degradation behavior of hydrogels formed by Michael-type addition reactions. Biomacromolecules 2005;6:290-301.

[32] Fairbanks BD, Schwartz MP, Bowman CN, Anseth KS. Photoinitiated polymerization of PEGdiacrylate with lithium phenyl-2,4,6-trimethylbenzoylphosphinate: polymerization rate and cytocompatibility. Biomaterials 2009;30:6702-7.

[33] Fairbanks BD, Schwartz MP, Halevi AE, Nuttelman CR, Bowman CN, Anseth KS. A versatile synthetic extracellular matrix mimic via thiol-norbornene photopolymerization. Adv Mater 2009;21:5005-10.

[34] Bryant SJ, Nuttelman CR, Anseth KS. Cytocompatibility of UV and visible light photoinitiating systems on cultured NIH/3T3 fibroblasts in vitro. J Biomater Sci Polym Ed 2000;11:439-57. 
[35] Williams CG, Malik AN, Kim TK, Manson PN, Elisseeff JH. Variable cytocompatibility of six cell lines with photoinitiators used for polymerizing hydrogels and cell encapsulation. Biomaterials 2005;26:1211-8.

[36] Hoyle CE, Bowman CN. Thiol-ene click chemistry. Angew Chem Int Ed Engl 2010;49:154073.

[37] Lin CC, Raza A, Shih H. PEG hydrogels formed by thiol-ene photo-click chemistry and their effect on the formation and recovery of insulin-secreting cell spheroids. Biomaterials 2011;32:9685-95.

[38] Fairbanks BD, Singh SP, Bowman CN, Anseth KS. Photodegradable, photoadaptable hydrogels via radical-mediated disulfide fragmentation reaction. Macromolecules 2011;44:2444-50.

[39] Schwartz MP, Fairbanks BD, Rogers RE, Rangarajan R, Zaman MH, Anseth KS. A synthetic strategy for mimicking the extracellular matrix provides new insight about tumor cell migration. Integr Biol 2010;2:32-40.

[40] Benton JA, Fairbanks BD, Anseth KS. Characterization of valvular interstitial cell function in three dimensional matrix metalloproteinase degradable PEG hydrogels. Biomaterials 2009;30:6593-603.

[41] Anderson SB, Lin CC, Kuntzler DV, Anseth KS. The performance of human mesenchymal stem cells encapsulated in cell-degradable polymer-peptide hydrogels. Biomaterials 2011;32:3564-74.

[42] Raza A, Lin CC. The influence of matrix degradation and functionality on cell survival and morphogenesis in PEG-based hydrogels. Macromol Biosci 2013;13:1048-58.

[43] Raza A, Ki CS, Lin CC. The influence of matrix properties on growth and morphogenesis of human pancreatic ductal epithelial cells in 3D. Biomaterials 2013;34:5117-27.

[44] Ki CS, Shih H, Lin CC. Effect of 3D matrix compositions on the efficacy of EGFR inhibition in pancreatic ductal adenocarcinoma cells. Biomacromolecules 2013;14:3017-26.

[45] Lin T-Y, Ki CS, Lin C-C. Manipulating hepatocellular carcinoma cell fate in orthogonally crosslinked hydrogels. Biomaterials 2014;35:6898-906.

[46] McKinnon DD, Kloxin AM, Anseth KS. Synthetic hydrogel platform for three-dimensional culture of embryonic stem cell-derived motor neurons. Biomater Sci 2013;1:460-9.

[47] Pattison DI, Davies MJ. Actions of ultraviolet light on cellular structures. EXS 2006:131-57.

[48] Shih H, Lin CC. Visible-light-mediated thiol-ene hydrogelation using eosin-Y as the only photoinitiator. Macromol Rapid Commun 2013;34:269-73.

[49] Rizzuto F, Spikes JD. The eorsin-sensitized photooxidation of substituted phenylalanines and tyrosines. Photochem Photobiol 1977;25:465-76.

[50] Jones G, II, Farahat CW, Oh C. Photoinduced electron transfer involving eosin-tryptophan conjugates. Long-lived radical pair states for systems incorporating aromatic amino acid side chains. J Phys Chem 1994;98:6906-9.

[51] Stull ND, Breite A, McCarthy R, Tersey SA, Mirmira RG. Mouse islet of Langerhans isolation using a combination of purified collagenase and neutral protease. J Vis Exp 2012.

[52] Shih H, Lin CC. Cross-linking and degradation of step-growth hydrogels formed by thiol-ene photoclick chemistry. Biomacromolecules 2012;13:2003-12. 
[53] Shih H, Fraser AK, Lin CC. Interfacial thiol-ene photoclick reactions for forming multilayer hydrogels. ACS Appl Mater Interfaces 2013;5:1673-80.

[54] Jones G, Feng Z, Oh C. Photoinduced electron transfer for an eosin-tyrosine conjugate. Activity of the tyrosinate anion in long range electron transfer in a protein-like polymer matrix. J Phys Chem 1995;99:3883-8.

[55] Creed D. The photophysics and photochemistry of the near-UV absorbing amino acids - II. Tyrosine and its simple derivatives. Photochem Photobiol 1984;39:563-75.

[56] Johansson U, Ria M, Åvall K, Dekki Shalaly N, Zaitsev SV, Berggren P-O, et al. Pancreatic islet survival and engraftment is promoted by culture on functionalized spider silk matrices. PLOS ONE 2015;10:e0130169.

[57] Otonkoski T, Banerjee M, Korsgren O, Thornell LE, Virtanen I. Unique basement membrane structure of human pancreatic islets: implications for $\beta$-cell growth and differentiation. Diabetes Obes Metab 2008;10:119-27.

[58] Armanet M, Wojtusciszyn A, Morel P, Parnaud G, Rousselle P, Sinigaglia C, et al. Regulated laminin-332 expression in human islets of Langerhans. FASEB J 2009;23:4046-55.

[59] Li Y, Cao X, Li L-X, Brubaker PL, Edlund H, Drucker DJ. $\beta$-Cell Pdx1 expression is essential for the glucoregulatory, proliferative, and cytoprotective actions of glucagon-like peptide-1. Diabetes 2005;54:482-91.

[60] Andrali Sreenath S, Sampley Megan L, Vanderford Nathan L, Özcan S. Glucose regulation of insulin gene expression in pancreatic $\beta$-cells. Biochem J 2008;415:1-10.

[61] Liu Z, Jeppesen PB, Gregersen S, Chen X, Hermansen K. Dose- and glucose-dependent effects of amino acids on insulin secretion from isolated mouse islets and clonal INS-1E betacells. Rev Diabet Stud 2009;5:232-44. 


\section{Table of content graphic}

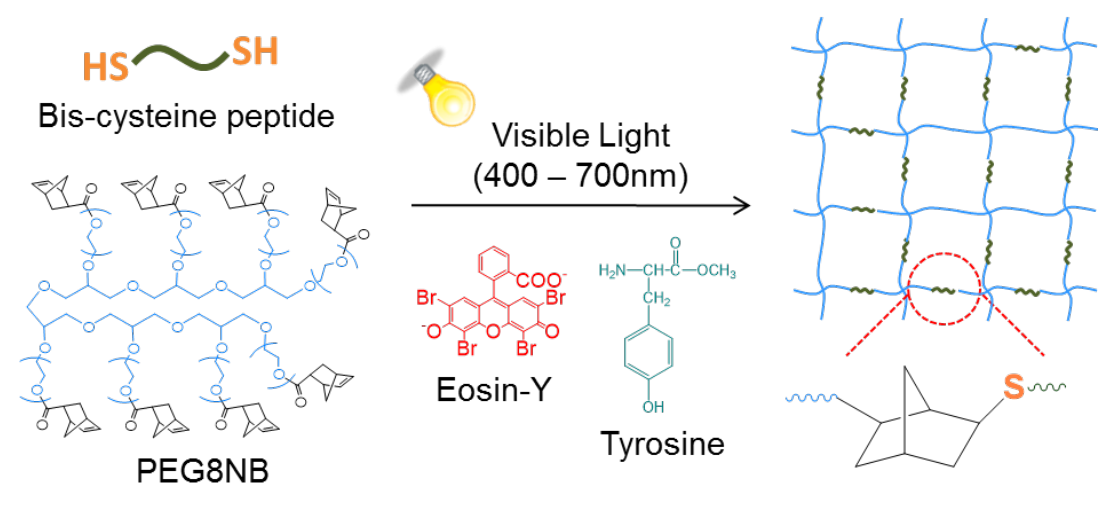

Biomimetic PEG-peptide hydrogel was developed through tyrosine-assisted visible-light thiolnorbornene crosslinking. Soluble tyrosine improves crosslinking and enhances cytocompatibility of the hydrogels. 